\title{
Sustained Morphine-Induced Sensitization and Loss of Diffuse Noxious Inhibitory Controls in Dura-Sensitive Medullary Dorsal Horn Neurons
}

\author{
Akiko Okada-Ogawa, ${ }^{1}$ Frank Porreca, ${ }^{2}$ and Ian D. Meng ${ }^{3}$ \\ ${ }^{1}$ Department of Oral Diagnosis, School of Dentistry, Nihon University, Kanda-Surugadai, Chiyoda-ku, Tokyo 101-8310, Japan, ${ }^{2}$ Department of \\ Pharmacology, College of Medicine, University of Arizona, Health Sciences Center, Tucson, Arizona 85724, and ${ }^{3}$ Department of Physiology, College of \\ Osteopathic Medicine, University of New England, Biddeford, Maine 04005
}

Overuse of medications used to treat migraine headache can produce a chronic daily headache, termed medication overuse headache $(\mathrm{MOH})$. Although "overuse" of opioids, triptans, and over-the-counter analgesics can all produce MOH, the neuronal mechanisms remain unknown. Headache pain is likely to be produced by stimulation of primary afferent neurons that innervate the intracranial vasculature and the resulting activation of medullary dorsal horn $(\mathrm{MDH})$ neurons. The present study compared the receptive field properties of MDH dura-sensitive neurons in rats treated with morphine to those given vehicle. Animals were implanted with osmotic minipumps or pellets for sustained subcutaneous administration of morphine or vehicle 6-7 d before recording from dura-sensitive neurons. Electrical and mechanical activation thresholds from the dura were significantly lower in chronic morphine-treated animals when compared to vehicle controls. In addition, sustained morphine increased the cutaneous receptive field sizes. The presence of diffuse noxious inhibitory controls (DNICs) was examined by placing the tail in $55^{\circ} \mathrm{C}$ water during concomitant noxious thermal stimulation of the cutaneous receptive field, usually located in the ophthalmic region. The DNIC stimulus produced significant inhibition of heat-evoked activity in vehicle- but not chronic morphine-treated animals. Inactivation of the rostral ventromedial medulla with $4 \%$ lidocaine reinstated DNICs in chronic morphine-treated animals. These results are consistent with studies demonstrating a loss of DNICs in patients that suffer from chronic daily headache and may partially explain why overuse of medication used to treat migraine can induce headaches.

\section{Introduction}

The overuse of medication to treat migraine headache can induce chronic daily headaches in some patients, representing a significant challenge to current migraine drug therapies (Dowson et al., 2005). Medication overuse headache $(\mathrm{MOH})$ can result from chronic use of a wide spectrum of migraine medications, including opiates, triptans, and over-the-counter drugs such as acetaminophen (Limmroth et al., 2002). Migraine sufferers are most at risk of developing $\mathrm{MOH}$, indicating that the cause appears to involve both genetic factors associated with migraine and neuroplastic changes induced by chronic drug exposure (Wilkinson et al., 2001; Bahra et al., 2003; Limmroth and Katsarava, 2004; Meng and Porreca, 2004).

In animals, chronic morphine treatment affects nociceptive systems in a manner that would be expected to alter the sensitivity of neurons involved in headache pain (Mao and Mayer, 2001;

Received July 24, 2009; revised 0ct. 6, 2009; accepted Nov. 2, 2009.

This work was supported by National Institute on Drug Abuse Grants K02DA018408 and R01DA014548 to I.D.M. We thank Dr. Glenn Stevenson for his statistical advice and Dr. Barbara Winterson for her helpful comments on an earlier version of this manuscript.

Correspondence should be addressed to Dr. lan D. Meng, Department of Physiology, University of New England, 11 Hills Beach Road, Biddeford, ME 04005. E-mail: imeng@une.edu.

DOI:10.1523/JNEUROSCI.3623-09.2009

Copyright $\odot 2009$ Society for Neuroscience ～0270-6474/09/2915828-08\$15.00/0
Ossipov et al., 2003). Sustained morphine exposure increases substance $\mathrm{P}$ and CGRP (calcitonin gene-related peptide) levels in the spinal cord dorsal horn and dorsal root ganglion cells in vivo and in culture (Ménard et al., 1995; Ma et al., 2000; Powell et al., 2000; Gardell et al., 2002; King et al., 2005). Furthermore, morphine exposure produces behavioral hypersensitivity to mechanical and thermal stimulation and sensitizes neurons recorded in deep laminae of the lumbar spinal cord dorsal horn (Mao et al., 1994; Célèrier et al., 2000, 2001; Vanderah et al., 2001; Laulin et al., 2002; King et al., 2005). Many of these changes induced by morphine exposure are dependent on descending facilitation from the rostral ventromedial medulla (RVM), which appears to be enhanced following chronic morphine (Vanderah et al., 2001; Gardell et al., 2002; Bie and Pan, 2005; Xie et al., 2005; Meng and Harasawa, 2007).

As the brainstem counterpart to the spinal cord dorsal horn, the medullary dorsal horn $(\mathrm{MDH})$ receives nociceptive input from primary afferent neurons that innervate orofacial regions and the intracranial blood vessels (Dubner and Bennett, 1983; Goadsby, 1997). In migraineurs, the trigeminal nociceptive pathway exhibits increased excitability. The present study compared the properties of dura-sensitive $\mathrm{MDH}$ neurons in rats following sustained morphine or saline exposure. Cutaneous receptive field properties and the sensitivity of the dura to mechanical and electrical stimulation were examined. In addition, we tested neurons 
Table 1. Thresholds for the activation of neurons by mechanical and electrical stimulation of the dura

\begin{tabular}{llll}
\hline & von Frey $(\mathrm{g})$ & $\mathrm{A}-\delta(\mathrm{mA})$ & C-fiber $(\mathrm{mA})$ \\
\hline Vehicle & $4.6 \pm 0.5(42)$ & $3.6 \pm 0.3(21)$ & $3.4 \pm 0.8(5)$ \\
Morphine & $2.7 \pm 0.4^{* *}(37)$ & $2.1 \pm 0.3^{* *}(30)$ & $2.3 \pm 0.4(9)$
\end{tabular}

Data are represented as mean \pm SEM. The numbers in parentheses indicate the number of neurons for each treatment group in which thresholds were determined. ${ }^{* *} p<0.01$ compared to vehicle-treated animals (Student's $t$ test)

A

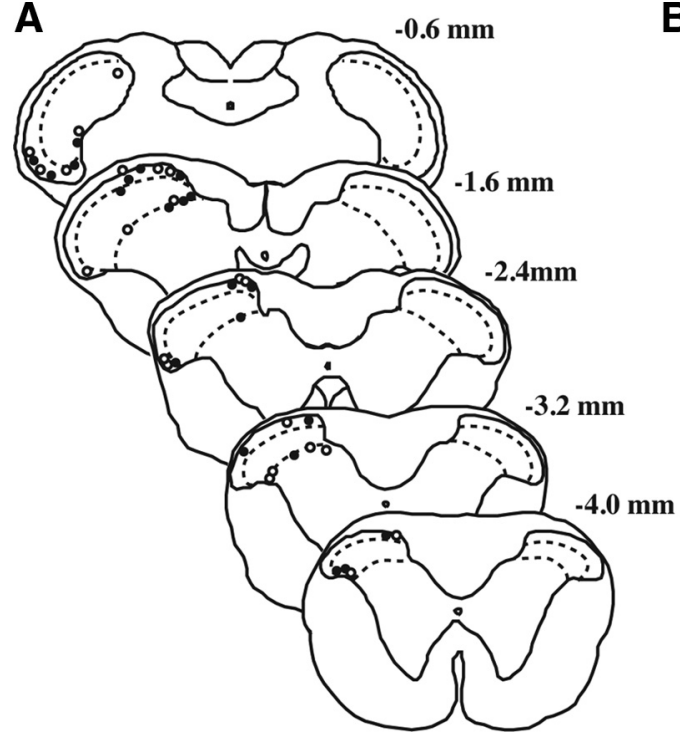

B

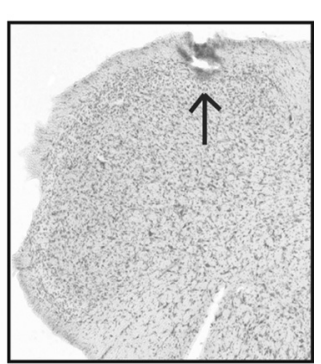

omy, animals were artificially respired and end-tidal $\mathrm{CO}_{2}$ was continuously monitored and maintained between 3.5 and $4.5 \%$. Body temperature was maintained at $37^{\circ} \mathrm{C}$ with a feedback-controlled heating pad. The jugular vein and femoral artery were cannulated for administration of drugs and measuring blood pressure, respectively. Mean arterial pressure and heart rate were monitored throughout the experiment. Fluids were replaced with a constant infusion of lactated Ringer's solution $(0.5 \mathrm{ml} / \mathrm{h})$. Rats were placed in a stereotaxic apparatus and prepared for $\mathrm{MDH}$ recording.

The dorsal brainstem was exposed and the dorsal C1 vertebral bone and part of the occipital bone removed to allow for insertion of a recording electrode. The brainstem was kept moist with warm mineral oil. A partial craniotomy was performed to expose the ipsilateral transverse sinus for electrical and mechanical stimulation of the dura. A side-by-side bipolar stimulating electrode made of platinum wire (separation $\sim 1.5 \mathrm{~mm}$ ) was positioned for electrical stimulation of the dura above the transverse sinus. Electrophysiological recording commenced $1 \mathrm{~h}$ after surgery.

Extracellular single-unit activity was recorded using tungsten electrodes (7-12 $\mathrm{M} \Omega$, FHC). The amplified, filtered signal was passed through a window discriminator and monitored with digital and storage oscilloscopes. Discriminated units triggered a digital oscilloscope to confirm constant spike shape and amplitude. Data were acquired using LabView (National Instruments) and analyzed off-line.

Characterization of neurons. Cutaneous brush stimulation of the ophthalmic division and brief periodic electrical stimulation (single shocks, 0.5-1.0 ms, 0.5-4 mA, $0.2 \mathrm{~Hz}$, delivered to the dural surface of the transverse sinus $\sim 3-5 \mathrm{~mm}$ from midline) were used as search stimuli. Neurons that responded to both electrical and mechanical stimulation of the dura were isolated for further study. Electrical, me-

for the effect of chronic morphine exposure on diffuse noxious inhibitory controls (DNICs) (Bouhassira et al., 1992; Bouhassira et al., 1993; Hernández et al., 1994; Villanueva and Le Bars, 1995). DNICs, which involve activation of descending inhibitory pathways from the subnucleus reticularis dorsalis, are impaired in chronic daily headache patients (Pielsticker et al., 2005). Our results indicate that sustained morphine exposure increases the sensitivity of dura-sensitive MDH neurons to both dura and cutaneous stimulation, and eliminates DNICs by activating pain facilitating neurons in the RVM.

\section{Materials and Methods}

General. Male Sprague Dawley rats were group housed (2-3/cage) in a climate-controlled environment with a $12 \mathrm{~h}$ light/dark cycle. Animals weighed 200-220 g at the time of implant surgery. All protocols were approved by the Committee on Animal Research at the University of New England, and animals were treated according to the policies and recommendations of the National Institutes of Health guidelines for the handling and use of laboratory animals.

Sustained morphine administration. All animals received a constant systemic exposure of either morphine or vehicle starting 6-7 d before and continuing throughout electrophysiological recordings. Morphine was administered by subcutaneous implants of two morphine sulfate pellets (75 mg each) or Alzet osmotic minipumps (model 2001) that released morphine sulfate $(64 \mathrm{mg} / \mathrm{ml})$ at a rate of $1 \mu \mathrm{l} / \mathrm{h}$ across $7 \mathrm{~d}$. Subcutaneous implantation of placebo pellets or a saline-filled osmotic minipump served as controls. Implants were performed under isoflurane anesthesia. In all studies the experimenter was blind to the treatment of the animal.

Electrophysiological recordings. Rats were anesthetized with urethane $(1.2 \mathrm{mg} / \mathrm{kg}$, i.p., with supplemental doses as necessary). After tracheot- chanical, and thermal stimulation were used to characterize each neuron. Cutaneous receptive fields were examined, first using innocuous mechanical stimulation and then noxious pressure and pinch (Meng et al., 1997). Neurons were classified as either low-threshold mechanoreceptive (LTM) units, wide dynamic range (WDR) units, or nociceptive-specific (NS) units. LTM units responded to hair movement and light touch and showed no increase in discharge with more intense stimuli. WDR units were sensitive to both non-noxious and noxious stimuli and showed an increase in discharge as the intensity of the stimulation increased. NS units were activated only by noxious stimuli applied to the cutaneous receptive field. Areas of brush (low-threshold) and pinch (highthreshold) receptive fields were mapped onto standard rat face outlines, scanned, and digitized for analysis.

Dural stimulation. Electrical thresholds for dura stimulation were determined by gradually increasing the intensity of stimulation until the lowest intensity that produced a response on at least $50 \%$ of the trials was reached. Mechanical receptive fields of the dura were mapped by stroking the dura with blunt forceps and indenting the dura with von Frey filaments (0.03-6.9 g, Stoelting). The region of highest sensitivity was used to determine the mechanical von Frey threshold. Using filaments of increasing strength applied to the dura for $2 \mathrm{~s}$, the response threshold was defined as the lowest intensity that evoked 1-2 spikes (Levy and Strassman, 2002a,b; Strassman and Levy, 2004).

Thermal stimulation. A standard sequence of thermal stimuli was applied twice to the center of the cutaneous receptive field using a contact thermode with a stimulating area of $25 \mathrm{~mm}^{2}$ (TSA, Medoc). Fast and slow heat ramps were used to activate $\mathrm{A}-\delta$ and $\mathrm{C}$ primary afferent neurons, respectively. Previous studies have indicated that a slow rate in rise of temperature $\left(0.9^{\circ} \mathrm{C} / \mathrm{s}\right)$ activated mostly $\mathrm{C}$-fibers, whereas a relatively fast rate $\left(9.0^{\circ} \mathrm{C} / \mathrm{s}\right)$ activated mostly A- $\delta$ primary afferents (Yeomans and 

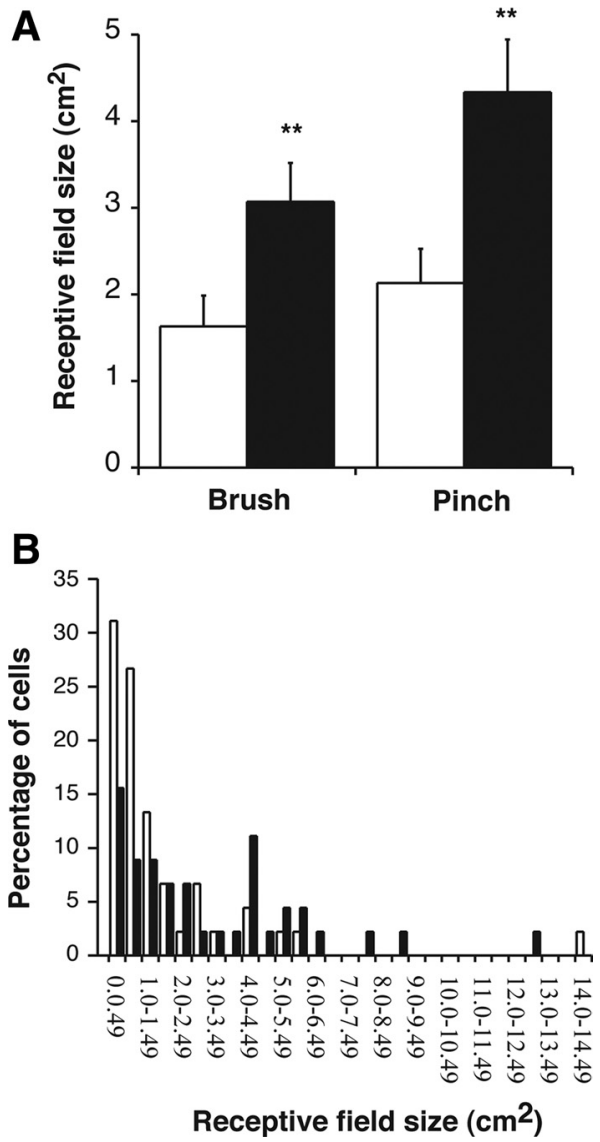

Figure 2. Cutaneous receptive field sizes. $\boldsymbol{A}$, Average brush and pinch cutaneous receptive field sizes were greater in morphine-treated animals (filled bars, $n=39$ ) when compared to vehicle-treated animals (open bars, $n=45$ ). $B$, A histogram of the brush receptive field sizes in vehicle- (open bars) and morphine- (filled bars) treated animals illustrating the distribution of receptive field sizes. ${ }^{* *} p<0.01$, Student's $t$ test.

Proudfit, 1996; Yeomans et al., 1996; Lu et al., 1997, 2004; Zachariou et al., 1997; Jones et al., 2003). Fast $\left(3.4^{\circ} \mathrm{C} / \mathrm{s}\right)$ and slow $\left(1.0^{\circ} \mathrm{C} / \mathrm{s}\right)$ heat ramps were used to increase temperatures from a $35^{\circ} \mathrm{C}$ holding temperature to a plateau of $52^{\circ} \mathrm{C}$. The plateau temperature was maintained for 10 and $5 \mathrm{~s}$ for the fast and slow heat ramps, respectively. In addition, $47^{\circ} \mathrm{C}$ stimulation $\left(3.4^{\circ} \mathrm{C} / \mathrm{s}\right.$ rate of rise) was applied for $10 \mathrm{~s}$. The sequence of $52^{\circ} \mathrm{C}$ (fast and slow heat ramps) and $47^{\circ} \mathrm{C}$ thermal stimulation was given with $3 \mathrm{~min}$ intervals between trials.

DNICs. The strength of DNICs was tested by placing the distal third of the tail in $55^{\circ} \mathrm{C}$ hot water for $10 \mathrm{~s}$ before and throughout the duration of a $52^{\circ} \mathrm{C}$ thermal stimulus applied to the center of the cutaneous receptive field. Activity evoked during the DNIC stimulus is compared with activity evoked during a control trial in which thermal stimulation is applied without placing the tail in hot water (Meng et al., 1997).

Microinjections into the RVM. In a separate set of experiments, DNICs were examined before and after microinjection of lidocaine (4\%,500 nl) into the RVM. A single 30 gauge stainless-steel microinjection cannula was inserted midline into the RVM ( -2.2 from bregma, $8.5 \mathrm{~mm}$ from surface of the cerebellum). The microinjection cannula was attached to a $50 \mu \mathrm{l}$ Hamilton microsyringe by PE-10 tubing, and infusions were conducted over a period of 3-5 min using a syringe pump. Movement of a small air bubble in the PE-10 tubing was monitored to ensure drug delivery (Manning et al., 2003; Harasawa et al., 2000; Meng and Johansen, 2004; Meng et al., 2005).

Data analysis. Low- and high-threshold receptive field areas were quantified by a planimetric method using National Institutes of Health Image software (version 1.61). Spontaneous activity was calculated by recording the average rate of activity (spikes per second) during the $10 \mathrm{~s}$ epoch immediately before the onset of each thermal stimulus. A total of
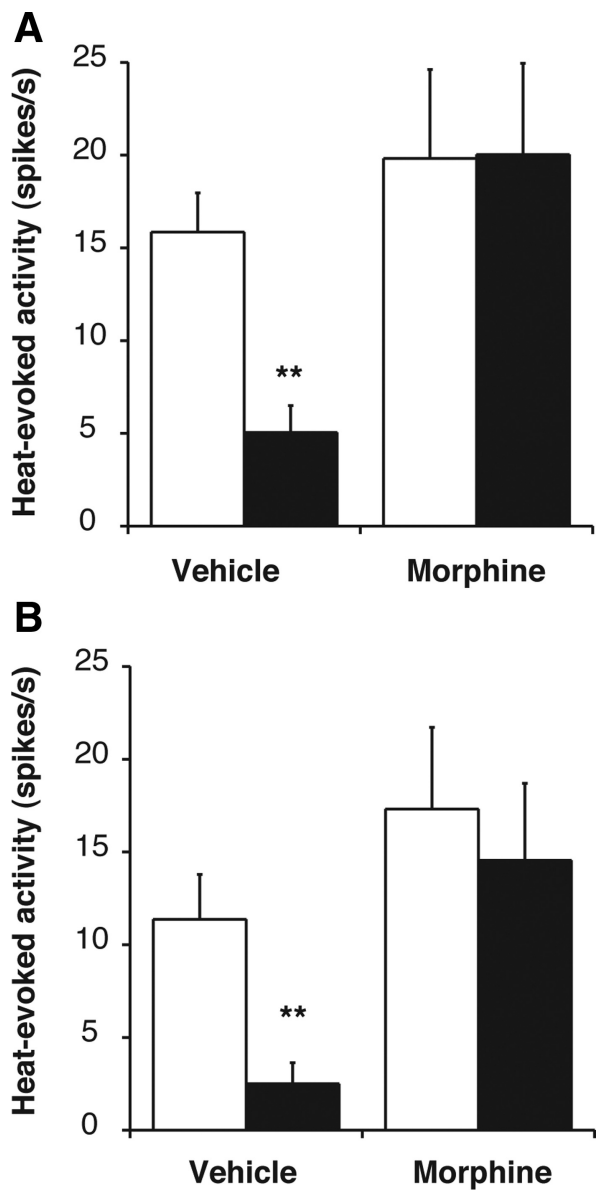

Figure 3. Diffuse noxious inhibitory controls in vehicle- and morphine-treated animals. No significant difference was seen between vehicle- and morphine-treated animals in fast $(\boldsymbol{A})$ and slow $(\boldsymbol{B})$ heat-evoked activity during the control stimulation trials (open bars). Heat applied to the cutaneous receptive field while the tail was place in $55^{\circ} \mathrm{C}$ water (filled bars) reduced neuronal activity only in vehicle-treated animals. ${ }^{* *} p<0.01$, two-way ANOVA with repeated measures.

six $10 \mathrm{~s}$ epochs, which corresponded to the presentation of the first six heat pulses, were averaged to calculate the spontaneous activity of a cell. The response to each heat pulse was calculated during the time of its peak temperature (Ogawa and Meng, 2009). All evoked responses were calculated by subtracting the spontaneous activity frequency recorded for $10 \mathrm{~s}$ immediately before the stimulus from the response elicited during the stimulus.

To calculate DNICs, group means for average evoked activity during the control trial was compared to activity evoked during the DNIC trial. A two-way ANOVA with repeated measures was used to compare data generated during control and DNIC stimulus trials for morphine- and vehicle-treated animals. Individual treatment comparisons used the Bonferroni post hoc test after ANOVA. All other group data were compared using either unpaired or paired Student's $t$ tests, depending on whether comparisons were between different drug treatment groups (morphine vs vehicle) or between different time points from the same drug treatment group. The D'Agostino-Pearson omnibus test was conducted on all datasets to test for normality. Variances were compared using the $F$ test (for two unpaired datasets) or Levene's test (for multiple datasets). All datasets conformed to a Gaussian distribution and did not have unequal variances. A Fisher exact probability test or $\chi^{2}$ test was performed to assess the frequencies of neurons encountered that responded to activation of different primary afferent fiber types. Data are presented as the mean \pm SEM, and differences were considered significant if $p<0.05$.

Histological verification. Electrolytic lesions were made at the end of each experiment to mark the recording electrode $(10 \mu \mathrm{A}, 10 \mathrm{~s})$ site. Rats 
were perfused with saline followed by $10 \%$ buffered formalin. The brain was removed and postfixed in $10 \%$ formalin overnight, followed by $30 \%$ sucrose. Coronal sections $(50 \mu \mathrm{m})$ were cut on a freezing microtome, mounted on slides, and stained with $0.3 \%$ cresyl violet.

\section{Results}

Data were collected from a total of 101 dura-sensitive neurons with WDR cutaneous receptive fields in 76 rats. Of these neurons, 48 were recorded in chronic morphine, and 53 were recorded from chronic vehicle-treated animals. Only WDR neurons were analyzed for this study, as inadequate numbers of LTM and NS neurons were sampled to make meaningful comparisons between treatment groups. Results obtained from neurons recorded in rats implanted with morphine or vehicle pellets $(n=41)$ and from neurons in those receiving constant infusion of morphine or saline through osmotic minipumps $(n=60)$ were combined in the overall analysis after it was determined that the results from each method of drug administration were not significantly different. For example, data presented in Table 1 indicates differences in mechanical and electrical stimulation thresholds in morphineand vehicle-treated animals. The dura von Frey thresholds in animals treated with morphine pellets $(2.9 \pm 0.6, n=16)$ were not statistically different from animals treated with morphine by osmotic minipumps $(2.6 \pm 0.5, n=21, p>0.05$, Student's $t$ test $)$. Likewise, thresholds to evoke A- $\delta$ activity (Table 1 ) were not different in animals treated with morphine via pellets (1990 \pm $469 \mathrm{~mA}, n=14$ ) or osmotic minipumps (2249 $\pm 454 \mathrm{~mA}, n=$ 16). In addition, two-tailed $F$ tests revealed no significant differences in the variances in the data generated from animals treated via pellets or minipumps $\left(F_{(16,21)}=1.79\right.$ and $F_{(17,22)}=$ 1.81 for von Frey and $\mathrm{A}-\delta$ thresholds, respectively; $p>0.05$ ). Reconstruction of the recording sites from electrolytic lesions in 43 animals with identified dura-sensitive neurons in both superficial (lamina I/II) and deep layers of the $\mathrm{MDH}$, ranging from regions just caudal to obex to $4.5 \mathrm{~mm}$ caudal to obex, is shown in Figure 1.

All characterized neurons responded to mechanical stimulation of the dura. Neurons recorded from morphine-treated animals had significantly lower mechanical stimulation thresholds when compared to vehicle-treated animals (Table 1$)(p<0.01$, Student's $t$ test). In addition, electrical stimulation thresholds were lower for A- $\delta$ evoked activity in chronic morphine-treated animals (Table 1) ( $p<0.01$, Student's $t$ test). A similar trend was seen in C-fiber evoked activity, although the difference did not reach statistical significance ( $p=0.2$, Student's $t$ test). Based on conduction velocities calculated from response latencies to electrical stimulation of the dura, the percentage of neurons receiving input from dural A- $\delta$ and/or $\mathrm{C}$ afferent fibers did not differ between treatment groups $\left(\chi^{2}=0.15, \mathrm{df}=2, p=0.93\right)$. Out of a total of 33 neurons tested in morphine-treated animals, 21 (64\%) received only A- $\delta$ input, $11(33 \%)$ received A- $\delta$ plus $C$ fiber input, and only $1(1 \%)$ received only $\mathrm{C}$ afferent fiber input. Of 34 neurons tested in vehicle control animals, $26(76 \%)$ received only $\mathrm{A}-\delta$ input, $5(15 \%)$ received $\mathrm{A}-\delta$ plus $\mathrm{C}$ fiber input, and $3(9 \%)$ received only $\mathrm{C}$ afferent fiber input.

Cutaneous fields were generally located in the region innervated by the ophthalmic branch of the trigeminal nerve. A comparison of the receptive field sizes revealed significantly larger cutaneous receptive fields in chronic morphine-treated animals (Fig. 2A,B). Sustained morphine increased the average size of both the brush-evoked and pinch-evoked cutaneous receptive fields ( $p<0.01$, Student's $t$ test). Perhaps the most telling sign of the receptive field expansion is the difference in the percentage of
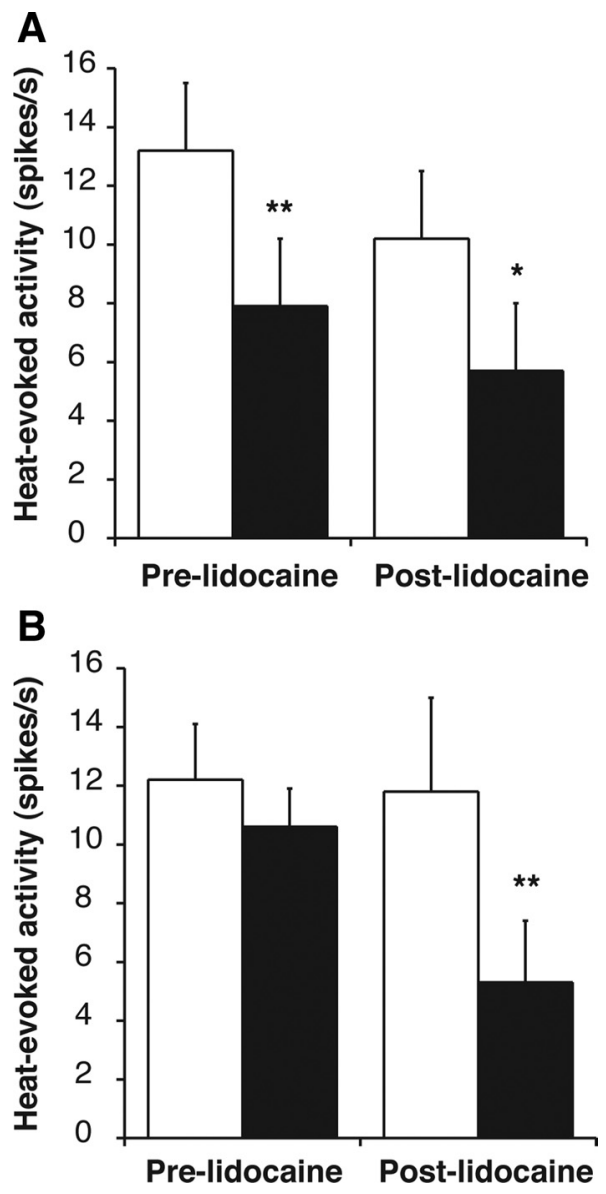

Figure 4. Microinjection of lidocaine into the RVM. $\boldsymbol{A}$, In vehicle-treated animals, the DNIC stimulus reduced heat-evoked activity both before and after injection of lidocaine into the RVM. $\boldsymbol{B}$, Morphine-treated animals did not demonstrate DNICs before lidocaine microinjections. However, after RVM lidocaine, the DNIC stimulus reduced heat-evoked activity in a manner similar to vehicle-treated animals. Open bars represent mean heat-evoked activity from control stimulation trials and filled bars represent mean heat-evoked activity while the tail was immersed in $55^{\circ} \mathrm{C}$ water. Data are for neuronal activity evoked by the fast heat ramp stimulus (see text for stimulus details). $n=9$ per treatment group; ${ }^{*} p<0.05,{ }^{* *} p<0.01$ versus the control stimulation trial, paired Student's $t$ test.

neurons with receptive fields that include part, or all, of the cornea. A total of 25 of 48 neurons in chronic morphine-treated animals had receptive fields that included the cornea, whereas only 10 of 53 neurons in control animals included corneal receptive fields. The difference in frequency of corneal receptive fields was highly significant between the two treatment groups $(p<$ 0.001 , Fisher's exact probability test).

Spontaneous activity did not differ between dura-responsive neurons recorded in morphine- $(1.2 \pm 0.6$ spikes/s $)$ and vehicle$(0.7 \pm 0.2$ spikes/s $)$ treated animals. In addition, activity evoked by both the slow and fast heat ramps during the control trials did not differ between morphine- $(n=17)$ and vehicle- $(n=16)$ treated animals (Fig. 3) (two-way ANOVA with the drug treatment effect insignificant for both fast, $F_{(1,31)}=3.53$, and slow, $F_{(1,31)}=4.09$, heat). However, the amount of inhibition produced by placing the tail in $55^{\circ} \mathrm{C}$ water was significantly greater in vehicle-treated animals (two-way ANOVA with a significant interaction between drug treatment and application of the DNIC stimulus for both fast, $F_{(1,31)}=10.88, p<0.01$, and slow, $F_{(1,31)}=$ $4.60, p<0.05$, heat). The inhibition produced by placement of the tail in hot water in vehicle-treated animals was entirely lost 
in morphine-treated animals, with comparable effects observed with both the fast heat- and slow heat-evoked responses (Fig. $3 A, B$ ).

In a subset of animals, the influence of the RVM on the loss of DNICs was examined by inactivating the RVM with microinjections of $4 \%$ lidocaine. As shown in Figure 4, in both the vehicle- and morphine-treated animals, RVM lidocaine did not affect noxious thermal stimulation-evoked activity during the control trials (i.e., in the absence of the DNIC stimulus; $p>0.05$, Student's $t$ test). In control animals, insertion of the tail into a hot water bath reduced noxious heat-evoked activity to $60.0 \pm 9.5 \%$ of control activity before RVM lidocaine and $56.4 \pm 16.4 \%$ of control activity after RVM lidocaine microinjections. In chronic morphine-treated animals, DNICs were not observed before lidocaine microinjections, as heat-evoked activity during the tail stimulation remained at 99.2 $\pm 12.3 \%$ of control. After RVM lidocaine, however, a complete restoration of DNICs was observed (Fig. 5), as activity was reduced to $48.8 \pm 12.3 \%$ of control. The values described above were calculated from activity evoked by the fast heat ramp stimulus. Similar results were observed for activity evoked by the slow heat ramp (data not shown).

\section{Discussion}

The present study was designed to examine the possibility that neuroplastic changes induced by prolonged drug exposure can lead to increased excitability of nociceptive neurons involved in headache pain (Meng and Porreca, 2004). The general receptive field properties of dura-sensitive neurons in control animals were similar to those previously reported by other investigators (Davis and Dostrovsky, 1988; Burstein et al., 1998; Schepelmann et al., 1999). Consistent with these previous studies, neurons had nociceptive cutaneous receptive fields located mainly in the periorbital region and received dural input from Aand C-fiber afferent neurons. The receptive field properties of neurons recorded from animals treated with sustained morphine administration, however, demonstrated significant differences when compared to control animals. In morphine-treated animals, cutaneous receptive field sizes were larger, activation thresholds to electrical and mechanical stimulation of the dura were lower, and inhibition of heat-evoked activity by a remote noxious stimulus (DNICs) was abolished. Finally, it was demonstrated that DNICs could be reinstated in morphine-treated animals by inactivating the RVM with lidocaine.

The increase in cutaneous receptive field sizes and the reduction in activation thresholds in dura-sensitive neurons following sustained morphine exposure are consistent with behavioral

\section{Pre-lidocaine control}

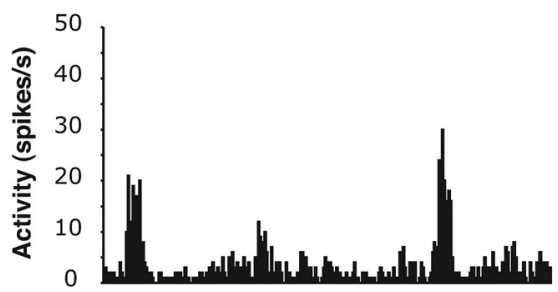

Pre-lidocaine DNIC

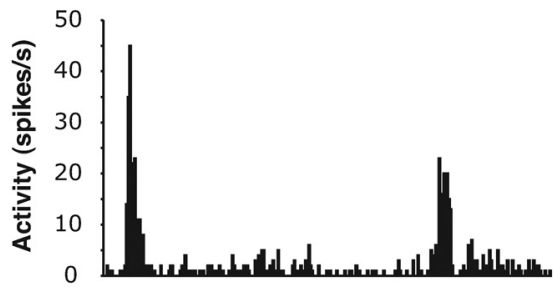

Post-lidocaine control

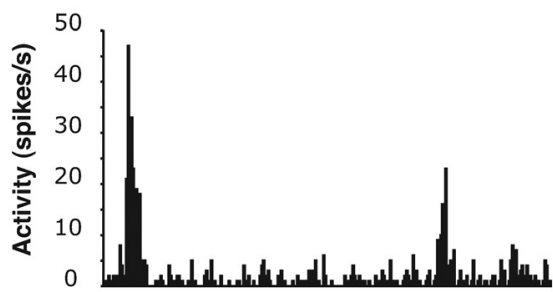

Post-lidocaine DNIC

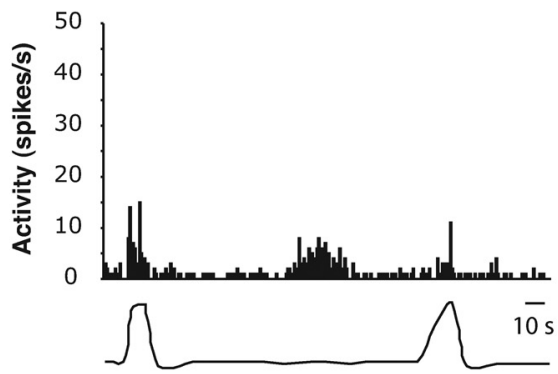

Fast heat $\left(52^{\circ} \mathrm{C}\right)$

Figure 5. Example neurons recorded from vehicle- (left) and morphine- (right) treated animals. Fast and slow heat ramps were used to evoke activity before and during immersion of the tail in $55^{\circ} \mathrm{C}$ water (DNIC stimulus). Left panels, In the vehicle-treated animal, the DNIC stimulus inhibited activity both before and after RVM lidocaine. Right panels, In the morphine-treated animal, trial remained unaffected $15 \mathrm{~min}$ after the lidocaine microinjection, whereas the DNIC stimulus now produced a profound decrease in heat-evoked activity. Bin width equals $1 \mathrm{~s}$.

studies that have demonstrated hypersensitivity to thermal and mechanical stimulation of the hindpaw and to mechanical stimulation of cephalic regions using identical morphine administration protocols (Kovelowski et al., 2000; Vanderah et al., 2000, 2001; Gardell et al., 2002; Meng and Porreca, 2004; Dogrul et al., 2005; King et al., 2005; Xie et al., 2005). In an apparent inconsistency, the present study revealed no differences in thermal stimulation-evoked activity in morphine-treated animals. However, increased behavioral sensitivity to thermal stimulation would likely involve a decrease in the activation threshold. Since neuronal activity was only assessed following a suprathreshold thermal stimulus, additional studies that perform a complete thermal stimulus-intensity response function would be necessary to enable the detection of decreased activation thresholds in response to sustained morphine exposure. 
The changes in receptive field properties observed in morphine-treated animals are similar those reported after application of inflammatory soup to the dura (Burstein et al., 1998). In this animal model of headache, application of inflammatory mediators to the dura leads to mechanical hypersensitivity to both cranial and extracranial regions as well as sensitization of both dural primary afferent and MDH neurons (Drummond, 1987; Strassman et al., 1996; Burstein et al., 1998; Burstein et al., 2000; Katsarava et al., 2002; Kaube et al., 2002; de Tommaso et al., 2003; Katsarava et al., 2003; Valeriani et al., 2003; Weissman-Fogel et al., 2003; Oshinsky and Gomonchareonsiri, 2007; Edelmayer et al., 2009). As with prolonged morphine exposure, inflammation of the dura increased cutaneous receptive field sizes and lowered dural activation thresholds. The sensitization of these neurons following inflammatory soup involved centrally mediated events, evidenced by its persistence after lidocaine application to the dura. Furthermore, similar to morphine-induced behavioral hypersensitivity, hypersensitivity following dural inflammation involves descending facilitation from the RVM (Edelmayer et al., 2009).

The RVM, which includes the nucleus raphe magnus and surrounding nucleus gigantocellularis pars $\alpha$, modulates nociceptive neurons though direct projections to the $\mathrm{MDH}$ (Porreca et al., 2002; Fields et al., 2005). Activation of one class of RVM neurons, the on-cell, facilitates nociceptive transmission, whereas activation of a second class of RVM neurons, the off-cell, inhibits nociceptive transmission. As mentioned, evidence implicates the activation of on-cells in behavioral hypersensitivity induced by both dural inflammatory soup application and sustained morphine exposure. Inactivation of the RVM attenuates allodynia produced by either dural inflammatory soup or morphine treatment (Vanderah et al., 2001; Gardell et al., 2002; Edelmayer et al., 2009). In addition, inflammatory soup applied to the dura activated RVM on-cells, while sustained morphine exposure increased the percentage of identified RVM on-cells (Meng and Harasawa, 2007; Edelmayer et al., 2009).

Although the present study used microinjections of lidocaine to inactivate all neurons (and fibers of passage), both morphine exposure and inflammation appear to increase the excitation of RVM pain facilitatory neurons. In vitro studies in RVM slice following chronic morphine or inflammation found increased glutamatergic inputs in subclasses of neurons thought to be involved in facilitating dorsal horn nociceptive transmission (Bie and Pan, 2005; Zhang and Hammond, 2009). The effect of specific glutamate receptor antagonists in the behavioral hypersensitivity produced by chronic morphine exposure has yet to be examined. However, acute systemic morphine analgesia does involve activation of glutamate receptors in the RVM (Heinricher et al., 1999, 2001).

Increased descending facilitation from the RVM also appears to be responsible for the loss of DNICs in morphine-treated animals. Inhibition produced by application of a remote noxious stimulus does not involve the RVM directly, as studies have shown that RVM lesions do not affect DNICs (Le Bars et al., 1992). Instead, the DNIC pathway includes afferent projections from the dorsal horn to the nucleus reticularis dorsalis (NRD), and descending projections from the NRD to the dorsal horn (Dickenson et al., 1980; Bouhassira et al., 1992; Villanueva and Le Bars, 1995; Le Bars, 2002). Although noxious stimulation activates pain inhibitory neurons from the NRD, it produces the opposite response in the RVM, activating pain facilitating oncells (Anderson et al., 1977; Hernández et al., 1994).
An increase in the activation of on-cells by morphine treatment may change the balance of descending inhibition and facilitation from the NRD and RVM, respectively. Under normal experimental conditions, remote noxious stimulation produces inhibition (DNICs), effectively masking descending facilitation from the RVM produced by on-cell activation. However, after sustained morphine exposure, increased facilitation from the RVM may counteract the inhibition produced by NRD activation. The demonstration that RVM inactivation can reinstate DNICs in morphine-treated animals supports such a hypothesis.

The present results have clear implications for the interpretation of some recent clinical studies that have demonstrated impaired DNICs in patients suffering from chronic tension type headache (Pielsticker et al., 2005), chronic migraine (de Tommaso et al., 2007), and fibromyalgia (Kosek and Hansson, 1997; Lautenbacher and Rollman, 1997). While the authors of these studies interpreted the results as suggestive of a loss of descending inhibition, our data indicates that an increase in descending facilitation is a more likely cause of the impaired DNICs. Thus, the impairment of DNICs in a clinical population and its absence in the present study after sustained morphine exposure helps to validate the use of chronic morphine for studying the mechanisms chronic daily headache and potentially other chronic pain disorders. Indeed, opiates are commonly used to treat migraine but, like other migraine treatments, carry the risk of producing $\mathrm{MOH}$ in patients. One study in particular assessed the development of chronic daily headache in patients taking opiates for reasons other than pain management (Wilkinson et al., 2001). Patients who had a previous history of episodic migraine developed chronic daily headache over the course of opiate treatment, while those with no history of migraine did not develop chronic daily headache. Another study found that migraine sufferers with a history of opiate use for the treatment of their migraines were unresponsive to headache therapy using COX1/2 inhibitors (Jakubowski et al., 2005).

In summary, the sensitization and loss of DNICs in durasensitive $\mathrm{MDH}$ neurons is consistent with clinical observations that overuse of medication to treat migraine headache can lead to chronic daily headaches. Additional studies are necessary to understand the mechanisms involved in the neuroplastic changes produced by chronic exposure to morphine and, potentially, other migraine medications.

\section{References}

Anderson SD, Basbaum AI, Fields HL (1977) Response of medullary raphe neurons to peripheral stimulation and to systemic opiates. Brain Res 123:363-368.

Bahra A, Walsh M, Menon S, Goadsby PJ (2003) Does chronic daily headache arise de novo in association with regular use of analgesics? Headache 43:179-190.

Bie B, Pan ZZ (2005) Increased glutamate synaptic transmission in the nucleus raphe magnus neurons from morphine-tolerant rats. Mol Pain 1:7.

Bouhassira D, Villanueva L, Bing Z, le Bars D (1992) Involvement of the subnucleus reticularis dorsalis in diffuse noxious inhibitory controls in the rat. Brain Res 595:353-357.

Bouhassira D, Chitour D, Villanueva L, Le Bars D (1993) Morphine and diffuse noxious inhibitory controls in the rat: effects of lesions of the rostral ventromedial medulla. Eur J Pharmacol 232:207-215.

Burstein R, Yamamura H, Malick A, Strassman AM (1998) Chemical stimulation of the intracranial dura induces enhanced responses to facial stimulation in brain stem trigeminal neurons. J Neurophysiol 79:964-982.

Burstein R, Cutrer MF, Yarnitsky D (2000) The development of cutaneous allodynia during a migraine attack: clinical evidence for the sequential recruitment of spinal and supraspinal nociceptive neurons in migraine. Brain 123:1703-1709.

Célèrier E, Rivat C, Jun Y, Laulin JP, Larcher A, Reynier P, Simonnet G 
(2000) Long-lasting hyperalgesia induced by fentanyl in rats: preventive effect of ketamine. Anesthesiology 92:465-472.

Célèrier E, Laulin JP, Corcuff JB, Le Moal M, Simonnet G (2001) Progressive enhancement of delayed hyperalgesia induced by repeated heroin administration: a sensitization process. J Neurosci 21:4074-4080.

Davis KD, Dostrovsky JO (1988) Responses of feline trigeminal spinal tract nucleus neurons to stimulation of the middle meningeal artery and sagittal sinus. J Neurophysiol 59:648-666.

de Tommaso M, Valeriani M, Guido M, Libro G, Specchio LM, Tonali P, Puca F (2003) Abnormal brain processing of cutaneous pain in patients with chronic migraine. Pain 101:25-32.

de Tommaso M, Sardaro M, Pecoraro C, Di Fruscolo O, Serpino C, Lamberti P, Livrea P (2007) Effects of the remote C fibres stimulation induced by capsaicin on the blink reflex in chronic migraine. Cephalalgia 27:881-890.

Dickenson AH, Le Bars D, Besson JM (1980) Diffuse noxious inhibitory controls (DNIC). Effects on trigeminal nucleus caudalis neurones in the rat. Brain Res 200:293-305.

Dogrul A, Bilsky EJ, Ossipov MH, Lai J, Porreca F (2005) Spinal L-type calcium channel blockade abolishes opioid-induced sensory hypersensitivity and antinociceptive tolerance. Anesth Analg 101:1730-1735.

Dowson AJ, Dodick DW, Limmroth V (2005) Medication overuse headache in patients with primary headache disorders: epidemiology, management and pathogenesis. CNS Drugs 19:483-497.

Drummond PD (1987) Scalp tenderness and sensitivity to pain in migraine and tension headache. Headache 27:45-50.

Dubner R, Bennett GJ (1983) Spinal and trigeminal mechanisms of nociception. Annu Rev Neurosci 6:381-418.

Edelmayer RM, Vanderah TW, Majuta L, Zhang ET, Fioravanti B, De Felice M, Chichorro JG, Ossipov MH, King T, Lai J, Kori SH, Nelsen AC, Cannon KE, Heinricher MM, Porreca F (2009) Medullary pain facilitating neurons mediate allodynia in headache-related pain. Ann Neurol 65:184-193.

Fields HL, Basbaum AI, Heinricher MM (2005) Central nervous system mechanisms of pain modulation, Ed 5. Edinburgh: Elsevier, Churchill Livingstone.

Gardell LR, Wang R, Burgess SE, Ossipov MH, Vanderah TW, Malan TP Jr, Lai J, Porreca F (2002) Sustained morphine exposure induces a spinal dynorphin-dependent enhancement of excitatory transmitter release from primary afferent fibers. J Neurosci 22:6747-6755.

Goadsby PJ (1997) Pathophysiology of migraine: a disease of the brain. In: Headache (Goadsby PJ, Silberstein SD, eds), pp 5-24. Oxford: Butterworth-Heinemann.

Harasawa I, Fields HL, Meng ID (2000) Delta opioid receptor mediated actions in the rostral ventromedial medulla on tail flick latency and nociceptive modulatory neurons. Pain 85:255-262.

Heinricher MM, McGaraughty S, Farr DA (1999) The role of excitatory amino acid transmission within the rostral ventromedial medulla in the antinociceptive actions of systemically administered morphine. Pain 81:57-65.

Heinricher MM, Schouten JC, Jobst EE (2001) Activation of brainstem $\mathrm{N}$-methyl-D-aspartate receptors is required for the analgesic actions of morphine given systemically. Pain 92:129-138.

Hernández N, Dmitrieva N, Vanegas H (1994) Medullary on-cell activity during tail-flick inhibition produced by heterotopic noxious stimulation. Pain 58:393-401.

Jakubowski M, Levy D, Goor-Aryeh I, Collins B, Bajwa Z, Burstein R (2005) Terminating migraine with allodynia and ongoing central sensitization using parenteral administration of COX1/COX2 inhibitors. Headache 45:850-861.

Jones TL, Sweitzer SM, Wilson SP, Yeomans DC (2003) Afferent fiberselective shift in opiate potency following targeted opioid receptor knockdown. Pain 106:365-371.

Katsarava Z, Lehnerdt G, Duda B, Ellrich J, Diener HC, Kaube H (2002) Sensitization of trigeminal nociception specific for migraine but not pain of sinusitis. Neurology 59:1450-1453.

Katsarava Z, Giffin N, Diener HC, Kaube H (2003) Abnormal habituation of 'nociceptive' blink reflex in migraine- evidence for increased excitability of trigeminal nociception. Cephalalgia 23:814-819.

Kaube H, Katsarava Z, Przywara S, Drepper J, Ellrich J, Diener HC (2002) Acute migraine headache: possible sensitization of neurons in the spinal trigeminal nucleus? Neurology 58:1234-1238.
King T, Gardell LR, Wang R, Vardanyan A, Ossipov MH, Malan TP Jr, Vanderah TW, Hunt SP, Hruby VJ, Lai J, Porreca F (2005) Role of NK-1 neurotransmission in opioid-induced hyperalgesia. Pain 116:276-288.

Kosek E, Hansson P (1997) Modulatory influence on somatosensory perception from vibration and heterotopic noxious conditioning stimulation (HNCS) in fibromyalgia patients and healthy subjects. Pain 70:41-51.

Kovelowski CJ, Ossipov MH, Sun H, Lai J, Malan TP, Porreca F (2000) Supraspinal cholecystokinin may drive tonic descending facilitation mechanisms to maintain neuropathic pain in the rat. Pain 87:265-273.

Laulin JP, Maurette P, Corcuff JB, Rivat C, Chauvin M, Simonnet G (2002) The role of ketamine in preventing fentanyl-induced hyperalgesia and subsequent acute morphine tolerance. Anesth Analg 94:1263-1269, table of contents.

Lautenbacher S, Rollman GB (1997) Possible deficiencies of pain modulation in fibromyalgia. Clin J Pain 13:189-196.

Le Bars D (2002) The whole body receptive field of dorsal horn multireceptive neurones. Brain Res Brain Res Rev 40:29-44.

Le Bars D, Villanueva L, Bouhassira D, Willer JC (1992) Diffuse noxious inhibitory controls (DNIC) in animals and in man. Patol Fiziol Eksp Ter $55-65$.

Levy D, Strassman AM (2002a) Distinct sensitizing effects of the cAMPPKA second messenger cascade on rat dural mechanonociceptors. J Physiol 538:483-493.

Levy D, Strassman AM (2002b) Mechanical response properties of A and C primary afferent neurons innervating the rat intracranial dura. J Neurophysiol 88:3021-3031.

Limmroth V, Katsarava Z (2004) Medication overuse headache. Curr Opin Neurol 17:301-306.

Limmroth V, Katsarava Z, Fritsche G, Przywara S, Diener HC (2002) Features of medication overuse headache following overuse of different acute headache drugs. Neurology 59:1011-1014.

Lu Y, Pirec V, Yeomans DC (1997) Differential antinociceptive effects of spinal opioids on foot-withdrawal responses evoked by C fibre or A delta nociceptor activation. Br J Pharmacol 121:1210-1216.

Lu Y, Sweitzer SM, Laurito CE, Yeomans DC (2004) Differential opioid inhibition of C- and A delta-fiber mediated thermonociception after stimulation of the nucleus raphe magnus. Anesth Analg 98:414-419, table of contents.

Ma W, Zheng WH, Kar S, Quirion R (2000) Morphine treatment induced calcitonin gene-related peptide and substance $\mathrm{P}$ increases in cultured dorsal root ganglion neurons. Neuroscience 99:529-539.

Manning BH, Martin WJ, Meng ID (2003) The rodent amygdala contributes to the production of cannabinoid-induced antinociception. Neuroscience 120:1157-1170.

Mao J, Mayer DJ (2001) Spinal cord neuroplasticity following repeated opioid exposure and its relation to pathological pain. Ann N Y Acad Sci 933:175-184.

Mao J, Price DD, Mayer DJ (1994) Thermal hyperalgesia in association with the development of morphine tolerance in rats: roles of excitatory amino acid receptors and protein kinase C. J Neurosci 14:2301-2312.

Ménard DP, van Rossum D, Kar S, Jolicoeur FB, Jhamandas K, Quirion R (1995) Tolerance to the antinociceptive properties of morphine in the rat spinal cord: alteration of calcitonin gene-related peptide-like immunostaining and receptor binding sites. J Pharmacol Exp Ther 273:887-894.

Meng ID, Harasawa I (2007) Chronic morphine exposure increases the proportion of on-cells in the rostral ventromedial medulla in rats. Life Sci 80:1915-1920.

Meng ID, Johansen JP (2004) Antinociception and modulation of rostral ventromedial medulla neuronal activity by local infusion of a cannabinoid receptor agonist. Neuroscience 124:685-693.

Meng ID, Porreca F (2004) Mechanisms of medication overuse headache. Headache Currents 1:47-54.

Meng ID, Hu JW, Benetti AP, Bereiter DA (1997) Encoding of corneal input in two distinct regions of the spinal trigeminal nucleus in the rat: cutaneous receptive field properties, responses to thermal and chemical stimulation, modulation by diffuse noxious inhibitory controls, and projections to the parabrachial area. J Neurophysiol 77:43-56.

Meng ID, Johansen JP, Harasawa I, Fields HL (2005) Kappa opioids inhibit physiologically identified medullary pain modulating neurons and reduce morphine antinociception. J Neurophysiol 93:1138-1144.

Ogawa A, Meng ID (2009) Differential effects of the cannabinoid receptor 
agonist, WIN 55,212-2, on lamina I and lamina V spinal trigeminal nucleus caudalis neurons. Pain 141:269-275.

Oshinsky ML, Gomonchareonsiri S (2007) Episodic dural stimulation in awake rats: a model for recurrent headache. Headache 47:1026-1036.

Ossipov MH, Lai J, Vanderah TW, Porreca F (2003) Induction of pain facilitation by sustained opioid exposure: relationship to opioid antinociceptive tolerance. Life Sci 73:783-800.

Pielsticker A, Haag G, Zaudig M, Lautenbacher S (2005) Impairment of pain inhibition in chronic tension-type headache. Pain 118:215-223.

Porreca F, Ossipov MH, Gebhart GF (2002) Chronic pain and medullary descending facilitation. Trends Neurosci 25:319-325.

Powell KJ, Ma W, Sutak M, Doods H, Quirion R, Jhamandas K (2000) Blockade and reversal of spinal morphine tolerance by peptide and nonpeptide calcitonin gene-related peptide receptor antagonists. Br J Pharmacol 131:875-884.

Schepelmann K, Ebersberger A, Pawlak M, Oppmann M, Messlinger K (1999) Response properties of trigeminal brain stem neurons with input from dura mater encephali in the rat. Neuroscience 90:543-554.

Strassman AM, Levy D (2004) The anti-migraine agent sumatriptan induces a calcium-dependent discharge in meningeal sensory neurons. Neuroreport 15:1409-1412.

Strassman AM, Raymond SA, Burstein R (1996) Sensitization of meningeal sensory neurons and the origin of headaches. Nature 384:560-564.

Valeriani M, de Tommaso M, Restuccia D, Le Pera D, Guido M, Iannetti GD, Libro G, Truini A, Di Trapani G, Puca F, Tonali P, Cruccu G (2003) Reduced habituation to experimental pain in migraine patients: a $\mathrm{CO}(2)$ laser evoked potential study. Pain 105:57-64.

Vanderah TW, Gardell LR, Burgess SE, Ibrahim M, Dogrul A, Zhong CM, Zhang ET, Malan TP Jr, Ossipov MH, Lai J, Porreca F (2000) Dynorphin promotes abnormal pain and spinal opioid antinociceptive tolerance. J Neurosci 20:7074-7079.
Vanderah TW, Suenaga NM, Ossipov MH, Malan TP Jr, Lai J, Porreca F (2001) Tonic descending facilitation from the rostral ventromedial medulla mediates opioid-induced abnormal pain and antinociceptive tolerance. J Neurosci 21:279-286.

Villanueva L, Le Bars D (1995) The activation of bulbo-spinal controls by peripheral nociceptive inputs: diffuse noxious inhibitory controls. Biol Res 28:113-125.

Weissman-Fogel I, Sprecher E, Granovsky Y, Yarnitsky D (2003) Repeated noxious stimulation of the skin enhances cutaneous pain perception of migraine patients in-between attacks: clinical evidence for continuous sub-threshold increase in membrane excitability of central trigeminovascular neurons. Pain 104:693-700.

Wilkinson SM, Becker WJ, Heine JA (2001) Opiate use to control bowel motility may induce chronic daily headache in patients with migraine. Headache 41:303-309.

Xie JY, Herman DS, Stiller CO, Gardell LR, Ossipov MH, Lai J, Porreca F, Vanderah TW (2005) Cholecystokinin in the rostral ventromedial medulla mediates opioid-induced hyperalgesia and antinociceptive tolerance. J Neurosci 25:409-416.

Yeomans DC, Proudfit HK (1996) Nociceptive responses to high and low rates of noxious cutaneous heating are mediated by different nociceptors in the rat: electrophysiological evidence. Pain 68:141-150.

Yeomans DC, Pirec V, Proudfit HK (1996) Nociceptive responses to high and low rates of noxious cutaneous heating are mediated by different nociceptors in the rat: behavioral evidence. Pain 68:133-140.

Zachariou V, Goldstein BD, Yeomans DC (1997) Low but not high rate noxious radiant skin heating evokes a capsaicin-sensitive increase in spinal cord dorsal horn release of substance P. Brain Res 752:143-150.

Zhang L, Hammond DL (2009) Substance P enhances excitatory synaptic transmission on spinally projecting neurons in the rostral ventromedial medulla after inflammatory injury. J Neurophysiol 102:1139-1151. 